\title{
A Control-Oriented Knock Intensity Estimator
}

\author{
Enrico Corti, Claudio Forte, Gian Marco Bianchi, and Lorenzo Zoffoli, University of Bologna
}

\begin{abstract}
The performance optimization of modern Spark Ignition engines is limited by knock occurrence: heavily downsized engines often are forced to work in the Knock-Limited Spark Advance (KLSA) range. Knock control systems monitor the combustion process, allowing to achieve a proper compromise between performance and reliability. Combustion monitoring is usually carried out by means of accelerometers or ion sensing systems, but recently the use of cylinder pressure sensors is also becoming frequent in motorsport applications. On the other hand, cylinder pressure signals are often available in the calibration stage, where SA feedback-control based on the pressure signal can be used to avoid damages to the engine during automatic calibration.

A predictive real-time combustion model could help optimizing engine performance, without exceeding the allowed knock severity. Several knock models are available in the literature: most of those proposed for real-time applications are single zone or two-zone models, grounded on more complex CFD simulations. However, since the knock phenomenon is influenced by several factors, the real-time determination of KLSA requires the model to be adapted to the engine actual behavior. The approach proposed in the present paper, is based on the constant adaptation of a two-zone model to measured cylinder pressure data: typical results of the indicating analysis, available cycle-by-cycle and cylinder-by-cylinder, are used as inputs for the model, with the aim of predicting KLSA for the current running condition, without exceeding the maximum allowed knock intensity.
\end{abstract}

The approach has been applied to indicating data referring to non-knocking, light and heavy knocking conditions, showing a good prediction capability.

\section{Introduction}

Pressure-based combustion controls are becoming common in automotive engines management systems: many work in the literature can be found regarding the use of cylinder pressure sensors for the control of combustion phase, for standard production diesel engines ([1-6]) and prototype engines with innovative combustion approaches, such as Homogeneous Charge Compression Ignition (HCCI) [7-9], Reactivity Controlled Compression Ignition (RCCI) [10], Low Temperature Combustion (LTC) [11-12], Gasoline Compression Ignition (GCI) [13-14]. Other works focus on different benefits that can be obtained from the use of the pressure signal, such as air charge and residual gas estimation [15-16], Air-Fuel Ratio (AFR) estimation [17-18], pollutant emissions estimation and control [19]. The feedback control of pressure sensors is certainly used in many motorsport applications, such as Formula 1, as it can be inferred by FIA delegate reports [20]. The management of control parameters based on the cylinder pressure information is also common during the engine development stage, especially in the calibration phase ([21-22]): one of the tasks performed during calibration thanks to cylinder pressure technology is knock diagnosis, that is typically carried out analyzing the high-frequency content of the signal ([23]). The assessment of knock intensity is usually obtained applying statistical analyses to large data samples, containing knock indexes referring to several hundredths of engine cycles. This means that not only the assessment is not predictive, but it requires many engine cycles sampled in knocking conditions, for the determination of knock severity. Thus, the evaluation of Knock Limited Spark Advance (KLSA) requires exceeding the acceptable statistical knock intensity threshold, which may lead to engine damages. A real-time combustion model using as inputs the indicating data, able to predict the knock limit before dangerous knock intensity conditions are reached, would be very useful during engine development stages. Furthermore, for those applications where the cylinder pressure signal is available on-board, the model would improve engine performance: KLSA may change, depending on the piston thermal history ([24]), and a real-time model using indicating data would allow tuning the Spark Advance setting according to the actual running condition. For example, KLSA value estimated by the model at the beginning of a straight in a track during a race would be higher, compared to that evaluated at the end of the straight.

Many real-time compatible knock models can be found in the literature: some of them are meant for pre-calibration [25], other for Software or Hardware in the Loop simulations [26]. The presented approach is focused on the real-time execution of the model, with particular attention to its adaptation to actual cylinder pressure data.

Although the methodology is grounded on the availability of cylinder pressure signals, it has been developed based on cycle-based data (e.g., MFB10, MFB50, MAPO, CHR net) only. On one hand, this approach can be applied when the engine control system has not direct access to cylinder pressure signals, but receives indicating data cycle-by-cycle from a Combustion Analysis System (CAS): this is what usually happens during engine calibration. On the other hand, it could enable the use of the same methodology when indicating data are estimated, based on different signals [27, $28,29]$. Necessary information on the pressure trace evolution will thus be inferred, based on synthetic combustion tracers, such as MFB10, MFB50 and CHRnet.

\section{Combustion Model}

A real-time knock control strategy aimed at preventing engine damages should ground on the following modelling features:

0 the use of easily accessible combustion information, i.e., synthetic cycle-based indicating data;

o low computational power requirements. The model should be in the form of analytical functions, with explicit integration of derivative equations;

o predictive of severe knocking events, to prevent the risk of damage;

o adaptive to the actual operating condition of the engine with a few tuning parameters; 
o inclusive of stochastic model variables, to face with the cycle by cycle variability of engine combustions.

Most of the knock models proposed in the literature [30] are based on the estimation of autoignition time by means of chemical (detailed or reduced [31]) kinetic sub-models, or on empirical correlations (Livengood and $\mathrm{Wu}[32]$ ).

Millo et Al. [33] proposed a Kinetics Fit model of knock, in which the phenomenological equations of the induction time are tuned against detailed chemistry simulations. In the present work, a two steps chemistry model is presented, following the main structure proposed by Millo et Al. The first step of the model makes an estimation of the autoignition time of the mixture, the second step reproduces the effects of abnormal combustions on the pressure signal.

The induction time of knock is based on the Arrhenius-type equation of three different regions of temperatures:

$\frac{1}{\tau}=\frac{1}{\tau_{1}+\tau_{2}}+\frac{1}{\tau_{3}}(1)$

Where $\tau_{1}$ and $\tau_{2}$ represent the induction times for low and intermediate level of temperatures, while $\tau_{3}$ relates to the higher one. In the actual implementation, each induction time has the following form:

$\tau_{i}=A_{i} p^{-n_{i}} \exp \left(\frac{B_{i}}{T_{u}}\right) \varphi^{x_{i}}$

Where $\mathrm{p}, \mathrm{T}_{\mathrm{u}}$ and $\varphi$ are pressure, temperature and equivalence ratio of the unburned mixture, while Ai, ni, Bi and xi are the constants referred to each level of temperature. Following the model structure of Millo et Al [33], these constants come from a fitting procedure on the results of a kinetic tabulated map obtained by means of Cantera code $[34,35]$.

The first knock tracer index $\mathrm{kn}_{\text {trac }}$ is calculated by the following integral over the compression and expansion strokes:

$k n_{\text {trac }}=\int_{I V C}^{E V O} \frac{d t}{\tau}$

$\mathrm{kn}_{\text {trac }}$ is based on the thermodynamic variables of unburned fresh mixture on a crank angle basis, whose evaluation was accomplished by means of few simplified hypotheses in the equation of conservation of energy.

The evolution of the overall combustion progress variable is synthetized by a linear fitting, passing through MFB10 and MFB50. This choice was driven by the needs of lowering the computational cost of interpolating a Wiebe function: Figure 1 shows that the accuracy of the linear model is good within MFB10 and MFB85. On one hand, the contribution to the knock tracer integral with mass of fuel burned lower than $10 \%$ is negligible, on the other hand, the most likely values of $\mathrm{X}_{\mathrm{b}}$ at knock onset are lower than $90 \%$.

The overall evolution of pressure on a crank angle basis comes from the explicit integration of the first law of thermodynamics applied to the whole mass in the chamber:

$\frac{d p}{d \vartheta}=\frac{1}{V}\left((k-1) \frac{d x_{b}}{d \vartheta} C H R_{n e t}-k p \frac{d V}{d \vartheta}\right)$

where $\mathrm{p}$ is the mean pressure in the chamber, $\mathrm{V}$ is the cylinder volume, CHR net is the net cumulative heat release of the cycle. 


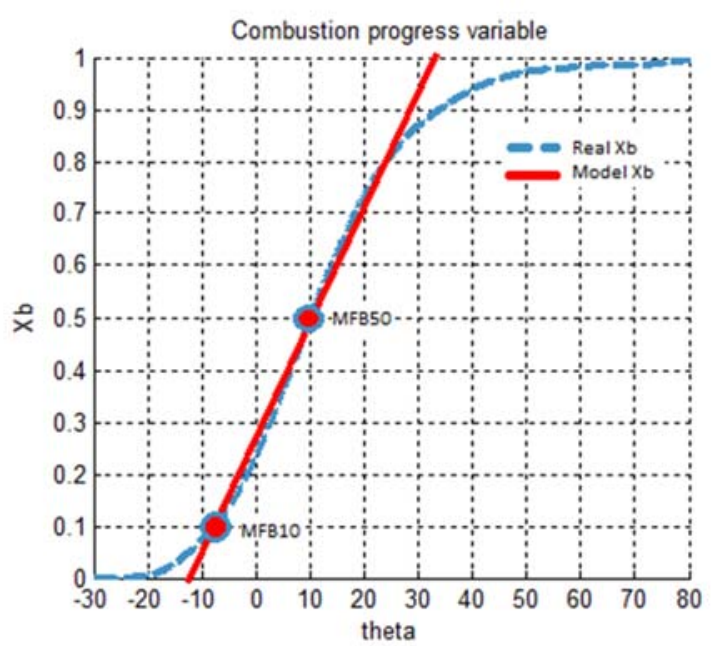

Figure 1: Combustion progress variable $\mathrm{Xb}$

Equation (4) is necessary if the control system implementing the KLSA estimation does not have access to the pressure samples, but only to cyclebased indicating parameters (namely, MFB10, MFB50, CHR $_{\text {net }}$ ): it represents the inversion of the same procedure used by the CAS system for the calculation of combustion variables, thus the estimation of pressure should be accurate, using the same $\mathrm{k}$ value. The pressure of the unburned mixture is supposed to be equal to the calculated mean value in the cylinder.

The estimation of the unburned temperature Tu comes from the integration of the energy balance of the unburned zone ([36-37]):

$\frac{d T_{u}}{d \vartheta}=\frac{1}{c_{p} m\left(1-X_{b}\right)}\left[\frac{1}{\omega} A_{u} h_{u}\left(T_{u}-T_{w}\right)+V_{u} \frac{d p}{d \vartheta}\right]$

Where $c_{p}$ is the specific heat capacity, $m$ the total mass in the cylinder, hu [33] is the heat transfer coefficient in the unburned zone, Au and $\mathrm{Vu}$ respectively the area and volume, $\omega$ engine speed. The integration of equation 5 is explicit and an estimation of the temperature $\mathrm{T}_{\mathrm{u}}$ at IVC (TIVC) is necessary: the initial Tu can be evaluated on a first attempt by means of the ideal gas law (based on the knowledge of cylinder pressure), or by results from 1D CFD simulations. Knock estimation is highly dependent on the identification of $\mathrm{T}_{\mathrm{IVC}}$, for this reason this starting temperature for equation 5 is subject to a tuning procedure for each cylinder and for every considered operating point, as it will be shown later in this work. kntrac can be calculated for each engine cycle, leading to the prediction of knock when its value exceeds 1.0.

The second step of the model is the identification of the effects on pressure signal when abnormal combustions occur. With the hypothesis [31] that all the unburned fuel at knock onset (i.e. kntrac reaches 1.0) suddenly burns, the estimation of pressure step caused by knock is:

$\Delta p_{\text {knock }}=\frac{(k-1)}{V}\left(\left(1-X_{b}\right) C H R_{n e t}\right)$

The knock index used for the identification of damage risk is finally defined as:

$k n_{\text {index }}=k_{1} \frac{r p m}{1000} k n_{\text {trac }}+k_{2} \max \left(0, k n_{\text {trac }}-1\right) \Delta p_{\text {knock }}$

Where $\mathrm{k}_{1}$ and $\mathrm{k}_{2}$ are model tuning constants. This definition of $\mathrm{kn}_{\text {index }}$ allows the model being directly comparable to the MAPO (Maximum Amplitude of Pressure Oscillations) [38], one of the most used indicating parameters for knock detection, defined as

$M A P O=\max \left(\left|p_{f i l t}\right|_{\theta_{s}}^{\theta_{e}}\right)$

The first term of equation (7), correlates the rate of combustion (represented by $\mathrm{kn}_{\text {trac }}$ ) to the pressure oscillation in case of normal combustions. This correlation (intrinsically included in the widely used MAPO thresholds definition of rpm/1000) accounts for the Arrhenius-like definition of kn trac Page 3 of 15 
and it is in line with the model suggested by Millo et Al. [33]. The second term of equation (7) allows adding the contribution of the pressure wave caused by knocking combustions.

Knock is a stochastic phenomenon and a real-time model should be able to represent both the variability of combustion and knocking events. In a previous work [38] the authors made an extensive use of CFD 3D analysis to reconstruct the correlations between variability of combustion and knock. Cycle by cycle instability is intrinsically included in the variability of the combustion angles (MFB10 and MFB50) used for the calculation of knock index. However, knock phenomenon is not solely identifiable by mean combustion angles: D'Adamo et Al [39] showed how a spatial distribution of temperature and mixture fraction clearly correlates with variability of knock events.

For this purpose, the unburned temperature Tu used in the integration of induction time is randomly chosen according to a normal distribution with standard deviation $\sigma(\mathrm{Tu})$ and a mean value predicted by means of equation (5). This deviation of temperature Tu from the mean value physically represents the probability of a spatial location to be in the last unburned zone of combustion. In the actual engine, the estimation of spatial distribution of $\mathrm{Tu}$ is $\sigma(\mathrm{Tu})=7 \mathrm{~K}$.

Figure 2 shows the evolution of the predicted and experimental MAPO indexes in two different engine operating conditions. Overall, the sensitivity of the model to combustion variability is good: the variability of temperature in the unburned zone allows better identifying the abrupt increase of MAPO with the fastest combustions. Once identified for significant running conditions, with different engine speed and knock intensities, the model constants $\mathrm{k}_{1}$ and $\mathrm{k}_{2}$ are kept constant for all the evaluations presented in this work.
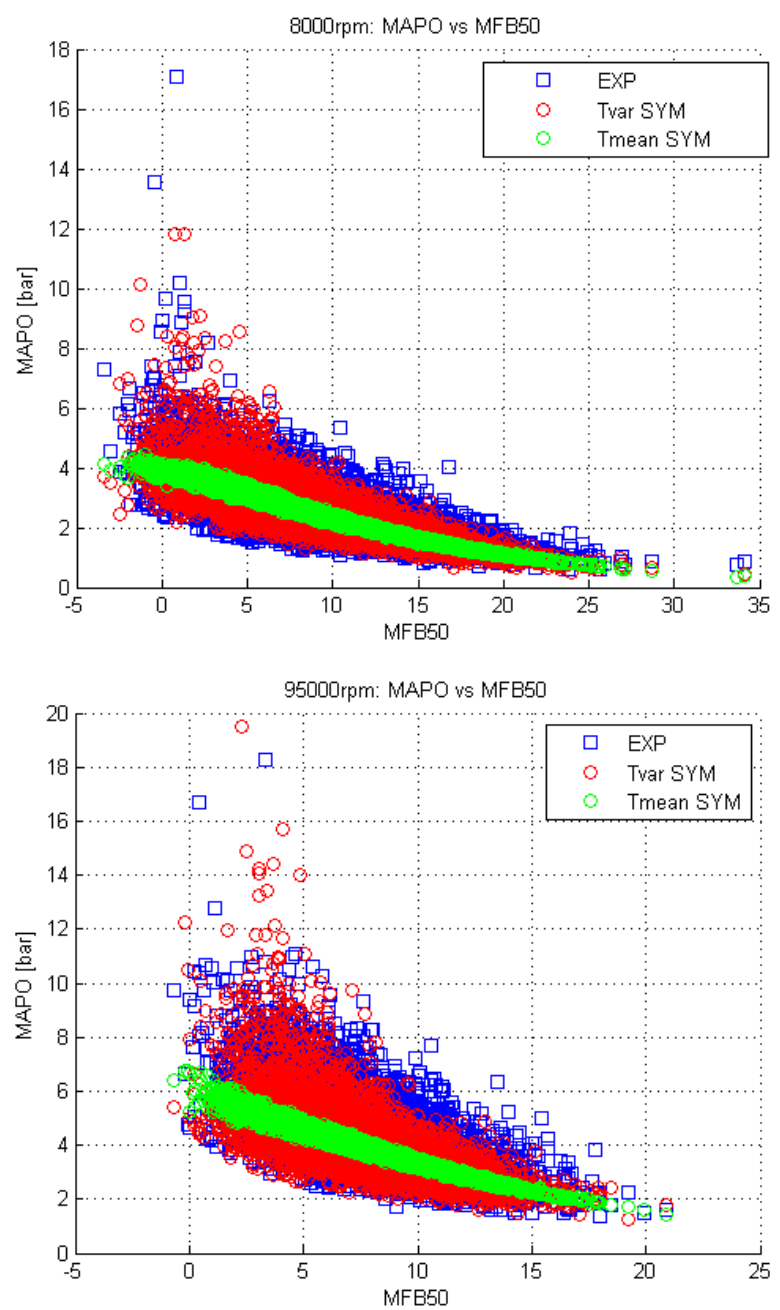

Figure 2: scatters of experimental and simulated MAPO versus MFB50

\section{Experimental Setup}

Page 4 of 15 
The engine under analysis is a Ducati-high performance unit, whose characteristics and running conditions are reported in Tab. 1. In order to increase the volumetric efficiency at high engine speed, a wide intake valve opening duration has been set, which makes the actual compression ratio lower than the geometric value. However, due to the ram and pressure waves tuning effects knocking combustions can happen even at high engine speed.

Table 1. Engine characteristics

\begin{tabular}{|l|l|}
\hline Displaced volume & $1198 \mathrm{cc}$ \\
\hline Bore x stroke & $106 \times 67.9 \mathrm{~mm}$ \\
\hline Connecting Rod & $122.5 \mathrm{~mm}$ \\
\hline Compression ratio & $12.8: 1$ \\
\hline Cylinders Configuration & $\mathrm{V} 2,90^{\circ}$ \\
\hline Intake Valve Opening & $18^{\circ} \mathrm{BTDC} \rightarrow 58^{\circ} \mathrm{ABDC}$ \\
\hline Exhaust Valve Opening & $58^{\circ} \mathrm{BBDC} \rightarrow 23^{\circ} \mathrm{ATDC}$ \\
\hline TEST CONDITIONS & \\
\hline Engine Speed & $5000,7000,8000,9500 \mathrm{rpm}$ \\
\hline Engine Load & WOT \\
\hline Lambda & 0,87 \\
\hline
\end{tabular}

The engine was tested on the test bench using Kistler 6052C32U20 cylinder pressure transducers and Alma Automotive Champion charge amplifier. An OBI-M2 Combustion Analysis System has been used to sample and analyse cylinder pressure, intake pressure, and actuations (coil charging and injection) signals. Pressure traces referring to more than 1000 engine cycles have been recorded for each engine operating point, for several different Spark Advance values. The signals are sampled @ 400kHz after a low-pass anti-aliasing filter (Butterworth, $3^{\text {rd }}$ order, $100 \mathrm{kHz}$ ). The evaluation of Indicated Mean Effective Pressure (IMEP), Rate of Heat Release (ROHR), Cumulative Heat Release (CHR) and therefore angle of Mass Fraction Burnt (MFB) has been carried out after a low-pass zero-delay $2^{\text {nd }}$ order Butterworth digital filter, with cut-off frequency set to $2.5 \mathrm{kHz}$. The evaluation of MAPO knock index has been obtained using a high-pass zero-delay $2^{\text {nd }}$ order Butterworth digital filter, with cut-off frequency set to 5 $\mathrm{kHz}$.

Although it would be possible to re-process sampled data off-line, the model and its application framework have been developed using the indicating parameters evaluated in real-time by the combustion analysis system. The CAS is able to send these data on the CAN bus, using a dedicated port, publishing them cylinder by cylinder, right at the end of each exhaust stroke, which allows closing the loop on the actuation (SA) within the next engine cycle. By feeding the knock simulation with the parameters evaluated in real-time it is possible to assess the actual performance attainable running the model in real-time using the feedback of the CAS.

\section{Real-time Model Adaptation to Indicating Data}

The Combustion Analysis System outputs several parameters for each cylinder and every engine cycle. These parameters may be used to feed the model, at the same time checking its ability to predict knock intensity. The model is then re-calibrated if predicted knock intensity is far from the measured value: the calibration is carried out only if measured or predicted knock intensity falls over a given threshold.

Since the simulated phenomenon is stochastic, a probabilistic analysis is applied to raw cycle-based knock intensity indexes (MAPO), so that the comparison of experimental and simulated series is carried out on a statistical basis. A common approach to the assessment of knock severity ([40]) is to use the MAPO intensity corresponding to a given probability: in this case the $99^{\text {th }}$ percentile has been selected.

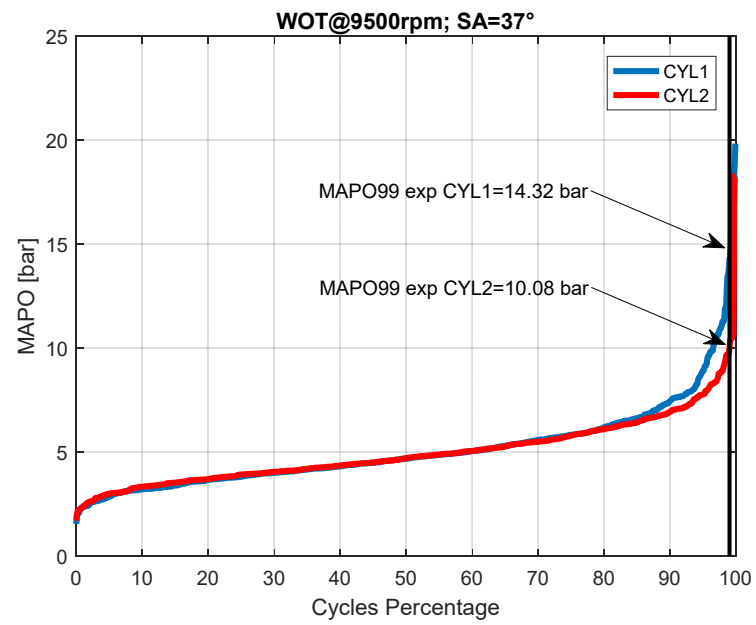

Figure 3: statistical knock indexes (MAPO99): experimental results 
Figure 3 shows how the statistical knock intensity index (MAPO99) is evaluated, sorting the elements (MAPO values pertaining to a given running condition and SA setting) in the buffer, and then selecting the value corresponding to the $99 \%$ position.

Since the model proved to predict the correct distribution (average value and scatter) of MAPO as a function of combustion phase, as already shown in Figure 2, if correctly calibrated and fed with experimental inputs (MFB10 and MFB50), the simulated MAPO distributions should be similar to the experimental, leading to a good prediction of $\mathrm{MAPO}_{99}$.
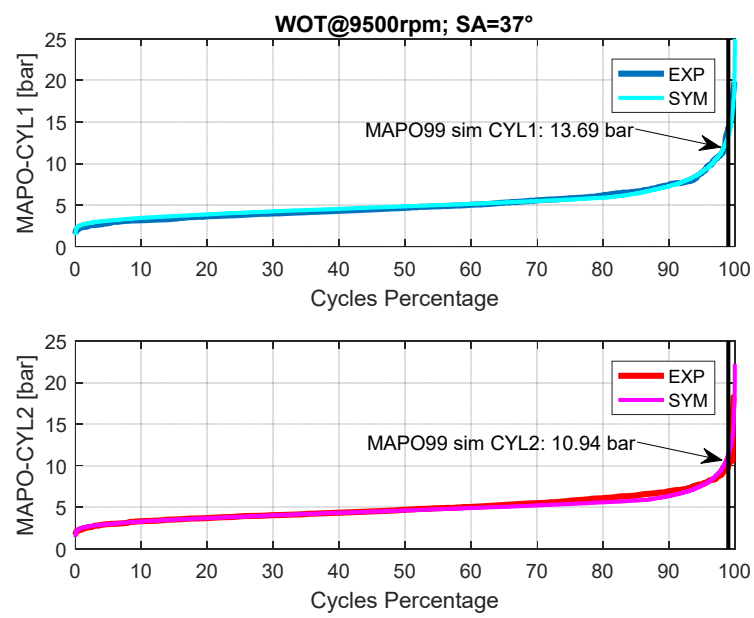

Figure 4: statistical knock indexes: experimental and simulated results

Figure 4 shows that the statistical distribution and the value of $\mathrm{MAPO}_{99}$ are correctly represented by the model: the simulation has been carried out coupling experimental results (MFB10 and MFB50) to a simulated temperature distribution. For each engine cycle the model has been executed using as inputs the experimental MFB10 and MFB50 values and ten different TIVC settings, randomly selected from a normal distribution. As explained in the previous section, IVC temperature distribution average value depends on engine speed and load, according to mono-dimensional CFD simulations, while the standard deviation is set to $7 \mathrm{~K}$.

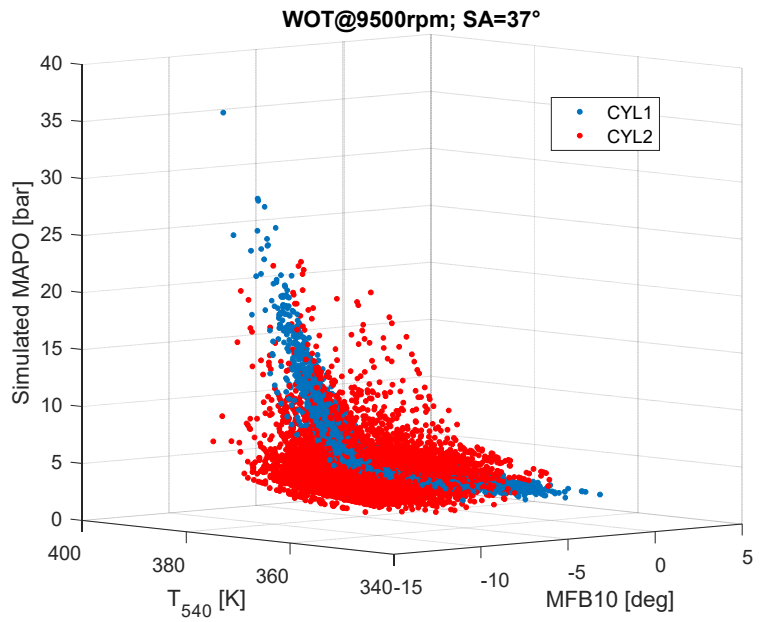

Figure 5: influence of experimental MFB10 and estimated TIVC on simulated MAPO

Figure 5 shows the influence of experimental MFB10 and estimated $\mathrm{T}_{\mathrm{IVC}}$ inputs on the model output (predicted MAPO).

This approach allows comparing simulated and experimental results, but it can also be used to predict the statistical knock intensity for a given SA setting, provided that the dependence of MFB10 and MFB50 distributions on SA can be assessed. In this case the model will use as inputs the estimated MFB10 and MFB50 distributions, together with the simulated temperature scatter, as shown previously. 
Many works in the literature [41-45] simulate cycle-to-cycle variations introducing normal distributions of MFB50: Figure 6 confirms that the approach is acceptable and also extendible to MFB10.
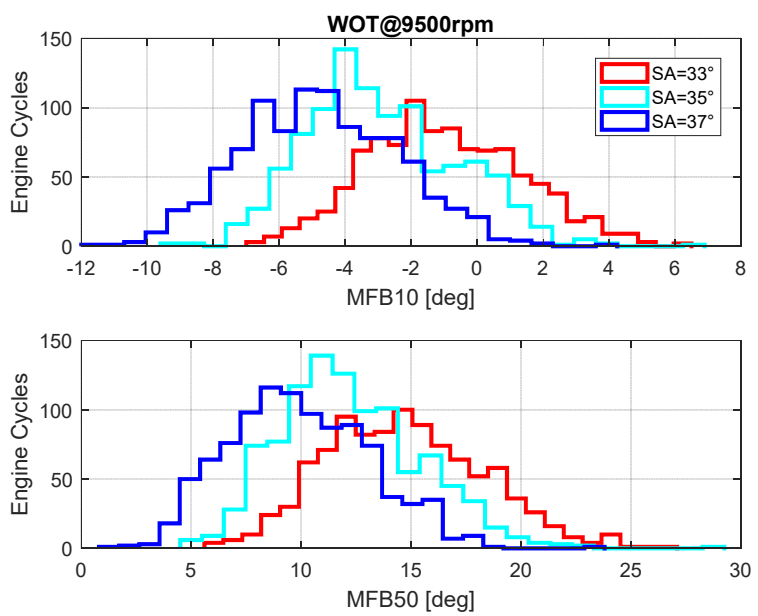

Figure 6: experimental MFB10 and MFB50 distributions

The fittings of MFB10 and MFB50 scatters with normal distributions show a linear dependence of the average value on spark advance, while standard deviation is substantially constant. It has to be remarked that the result is achieved thanks to the use of low-percentage Mass Fraction Burnt parameters: the extrapolation of the Mass Fraction Burnt introduced in the model description section allows using MFB50 instead of MFB85. The values of MFB85 may be influenced by the amount of heat transferred to the walls, and also by the way Cumulative Heat Release is calculated by the CAS: MFB85 evaluations based on net CHR (as in this case) may lead to anomalous distributions, especially in knocking conditions.
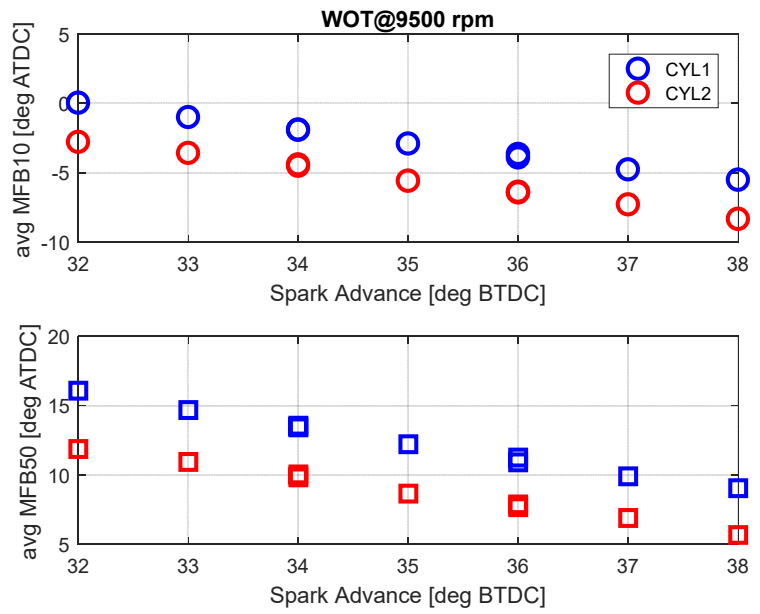

Figure 7: experimental MFB10 and MFB50 average values for different SA settings

Figure 7 shows that the variation of MFB10 with SA is linear, with very similar trends for the two cylinders (1 degree reduction in MFB10 for every degree increase of SA); the same behaviour can be observed for MFB50.

As regards the distributions standard deviation, it may be considered independent of Spark Advance both for MFB10 and MFB50 even if with different values, as it can be noticed in figure 8 . 

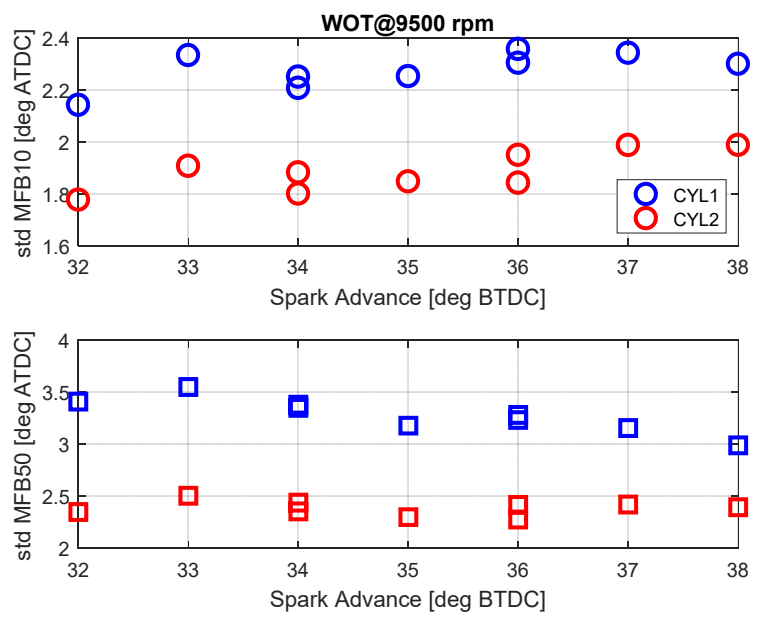

Figure 8: experimental MFB10 and MFB50 standard deviations for different SA settings

As stated previously, the use of the model for the prediction of statistical knock intensity for values of SA not yet explored experimentally requires the estimation of MFB10, MFB50 and TIVC distributions: the first two can be determined based on MFB-SA dependence evaluated experimentally with lower SA settings. For example, Figure 7 shows that, based on MFB10 and MFB50 experimental average values determined with SA=32 ${ }^{\circ}$ and $\mathrm{SA}=33^{\circ}$ it would be possible to extrapolate for higher SA values. As regards standard deviation, Figure 8 shows that once evaluated for low SA settings (e.g., $\mathrm{SA}=32^{\circ}$ or $33^{\circ}$ ) it can be considered constant for more advanced combustions. The model inputs required to predict knock intensity in advanced conditions can thus be inferred by the engine behavior in retarded conditions.

As already mentioned, the estimation of temperature distribution is carried out by means of a speed-dependent average value, and a fixed standard deviation: both values have been validated during the model calibration phase, leading to satisfying results in terms of scatter in the plane MAPOMFB50, as shown in Figure 2. However, the model sensitivity to TIVC is very high, and an estimation error of a few degrees may lead to significant errors in the prediction of MAPO99. Therefore, the value of temperature should be adapted to actual engine indicating data; the approach used in this work is organized as follows: every time a buffer of engine cycles pertaining to a given Spark Advance setting is completed, the experimental and simulated statistical knock indexes are evaluated and compared. If one of the two indexes is higher than a given threshold (meaning that the experimental or simulated knock index is approaching knocking levels) and the two values are significantly different, TIVC is adjusted, to minimize the prediction error.

Once MFB10, MFB50 and TIVC average values and standard deviations are available, the simulated MAPO99 is easily obtainable running the model. Since MFB10 and MFB50 are well correlated (with correlation coefficients in the range of 0.965-0.990 for the considered experimental data), the simulation is always carried out with values of MFB10 and MFB50 corresponding to the same probability (same value of the respective cumulative density functions). In the followings the general term 'MFB distributions' will be used, referring to both MFB10 and MFB50: even if the distributions are actually different (average values and standard deviations are different, as shown in figures 7 and 8), the two variables are considered related. The temperature at IVC, instead, is considered an independent variable with respect to MFB: the MAPO distribution corresponding to a given running condition can thus be determined combining the inputs of the model according to two independent sets of normal probability density functions. The first one is referred to the two related MFB10 and MFB50 distributions, the other refers to Tivc.

The number of evaluations necessary to complete a significant statistical test, resulting in a reliable MAPO99 value, however, could be excessively high for a real-time use of the model. The focus of the methodology is on the evaluation of MAPO99: this parameter represents the minimum knock intensity reached by the $1 \%$ higher intensity cycles. The simulated intensity can be high both in the case of high temperature (TIVC) or advanced combustion phase (MFB10, MFB50). The probability of achieving a given MAPO value (model output) is related to the probability of running the model with given values of temperature and combustion phase. 


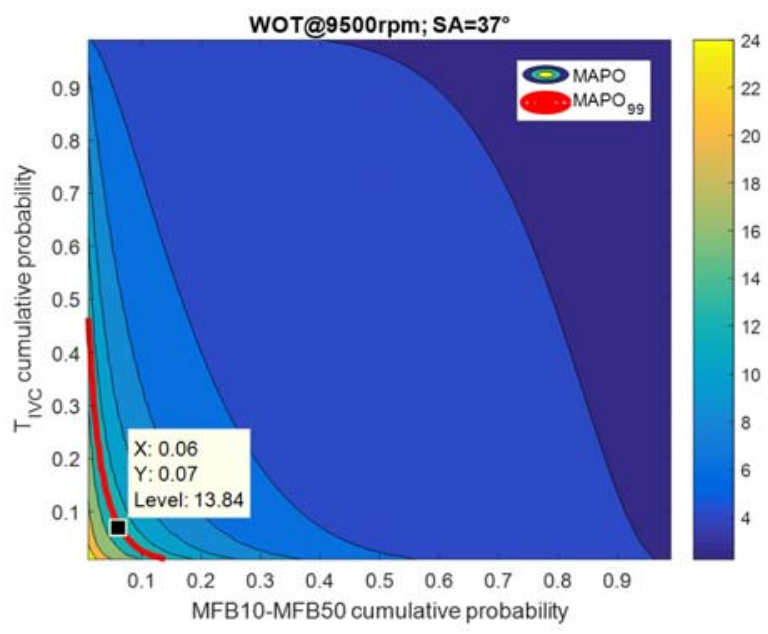

Figure 9: MAPO intensity as a function of TIVC and MFB10 (MFB50) cumulative probability

Figure 9 shows knock intensity as a function of the cumulative probability referred to TIVC and MFB distributions: the highlighted trace marks the transition through $\mathrm{MAPO}_{99}$ level for the considered simulation. All the cumulative probability combinations on the highlighted border are candidates for the representation of $\mathrm{MAPO}_{99}$ value. A very quick approach to the determination of $\mathrm{MAPO}_{99}$ consists in running the model only for a set of inputs (TIVC-99, MFB1099, MFB5099), defined picking the cumulative probability levels from the MAPO 99 curve highlighted in Figure 9 . Since the extreme values of the curve (intersection with X and Y axes) may be influenced by the engine running conditions (namely, by MFB10 and MFB50 standard deviation), an intermediate choice is more appropriate. In this case the setting guaranteeing the most similar values of MFB and TIVC cumulative probability $\left(\mathrm{P}_{\mathrm{MFB}}=6 \%\right.$ and $\mathrm{P}_{\mathrm{TIVC}}=7 \%$, respectively) have been selected.

The choice of running the simulation for a given pair of combustion phase and Inlet Valve Closing temperature pertains to the evaluation of MAPO 99 for unexplored SA settings: for the actual running conditions the simulation can be carried out on all the available engine cycles, as it has been done for the data reported in Figure 4. This operation is crucial for the model on-line calibration: on one hand, simulated MAPO 99 can be compared with the experimental result. In the case of a large discrepancy $\mathrm{T}_{\mathrm{IVC}}$ average value will be changed, reducing the prediction error. On the other hand, the actual $\mathrm{MAPO}_{99}$ value can be compared with that evaluated using the simplified approach (i.e., evaluating the model output with $\mathrm{T}_{\mathrm{IVC}}$ and MFB inputs corresponding to $7 \%$ and $6 \%$ cumulative probability): the probability settings can be adapted to reduce the discrepancy in the evaluation of MAPO 99 .

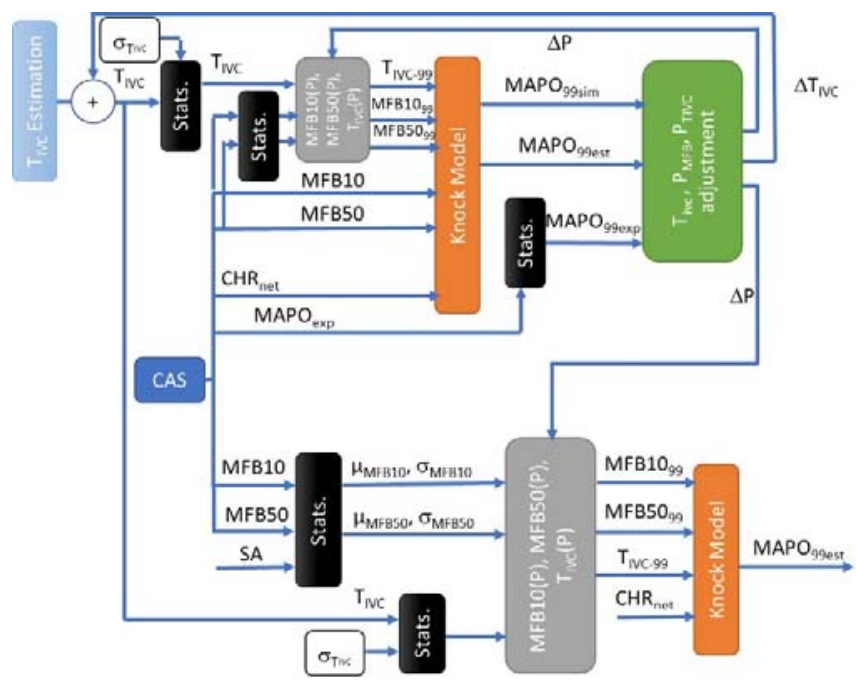

Figure 10: knock intensity estimation algorithm flow-chart

Figure 10 summarizes the flow of operations necessary to estimate the statistical knock index (MAPO99est) for a given SA setting. The knock model is fed with information from the CAS, and with estimated TIVC: input data refer to a statistical population of engine cycles, pertaining to the same running condition (safe SA setting). The model simulates knock intensity values corresponding to measured MFB10 and MFB50 distributions, and to a set of $\mathrm{T}_{\mathrm{IVC}}$ inputs, selected from the temperature normal probability density function. As already remarked, the pdf is determined thanks to a first attempt mean value (output of a lookup table), and a standard deviation set to $7 \mathrm{~K}$. The experimental and simulated MAPO distributions can then be compared: the difference between the simulated and experimental statistical knock index (MAPO99) for the current SA setting is used to tune TIVC mean value, provided that experimental or simulated $\mathrm{MAPO}_{99}$ exceeds a threshold. The adjustment of $\mathrm{T}_{\mathrm{IVC}}$ and MFB percentiles ( $\mathrm{P}_{\mathrm{TIVC}}$ and $\mathrm{P}_{\mathrm{MFB}}$ ) used for the simplified approach enabling the estimation of $\mathrm{MAPO}_{99}$ for a given SA, is based on the same data. MAPO 99 evaluated on current

Page 9 of 15 
indicating data is compared to that evaluated by the knock model, using as inputs MFB1099, MFB5099 and TIVC99 values picked for a given percentile (e.g., $6 \%$ and $7 \%$, respectively) from the respective distributions: the difference is used to tune percentiles values.

The determination of knock intensity corresponding to a given SA setting (lower part of the block diagram in Figure 10) is carried out using expected MFB10 and MFB50 distributions, obtained estimating the variation of mean values as a function of SA, under the hypothesis of constant standard deviation. The model is fed with adjusted percentiles, both for MFB and TIVC, and it outputs the estimated MAPO99. KLSA can be determined comparing the statistical knock index estimated by the model with a threshold: since the estimation is carried out only for a single set of MFB and TIVC values for each considered SA setting, the operation can be repeated several times, in order to assess KLSA, i.e., the value of SA leading to exceed the threshold.

The knock intensity estimator methodology proceeds according to the following steps:

1. The engine is operated in a knock-safe condition: a statistically significant population of indicating parameters (MFB10, MFB50, MAPO) is collected;

2. The model is run for each cycle available in the collected population, and the results (MAPO99) are compared to experimental data; statistical parameters for the prediction of knock intensity are adjusted. If the experimental or simulated knock intensities exceed a threshold and their values are significantly different, TIVC is adjusted;

3. A prediction of knock intensity is carried out for different SA settings, leading to the determination of KLSA. The model is run only once per SA setting, using the simplified determination of $\mathrm{MAPO}_{99}$.

The adaptive setting of $\mathrm{T}_{\mathrm{IVC}}$ and percentiles adapt the model outputs to different engine working conditions (changes in fuel characteristics, intake air, oil and water temperature, etc.) reducing at the same time the model calibration effort. Apart from TIVC mean value, and the percentiles PMFB and PTIVC, that can be adapted on-line, the parameters that need to be calibrated are TIVC standard deviation (set to $7 \mathrm{~K}$ ) and the three sets of parameters necessary to determine the induction times (eq. 2). These parameters, however, have been easily tuned starting from values found in the literature: the tuning has been carried out for non-knocking, light-knocking and heavy-knocking conditions, in a wide range of engine speed.

The methodology has been applied to steady state data, aiming at the estimation of KLSA for the given (steady) running conditions, using data referred to retarded combustions. The application in transient conditions would be possible, since the model could be run cycle-by-cycle, but a major change should be made to the statistical approach, which is grounded on the hypothesis of steady state conditions.

The computational power required by the knock intensity estimator is tightly tied to the way it is implemented: the model could be run cycle-bycycle, or only once the statistical buffer contains several hundredths engine cycles with the same SA setting. The second approach is typically applied during engine calibration: in this case the model runs on a real-time computer, with high computational power. The processing of 2000 engine cycles (on a i7 processor) in this case requires less than 0.1 seconds: obviously, the use of large buffers implies the need for a large amount of memory. These considerations must be taken into account when implementing the methodology on low-computational power and low-memory platforms (e.g., ECUs).

\section{Results}

The model-based knock intensity estimator has been applied off-line to engine data: it has to be remarked that, even if the estimator has not been implemented in a real time environment (in a Rapid Control Prototyping unit, or integrated in the CAS), the methodology has been developed focusing on the reduction of computational power.

The model has been run as described in the previous section, starting from low SA values, checking the predicted KLSA limit, and finally comparing this result with the value calculated on experimental data referring to the conditions where SA=KLSA. Obviously, the quality of the approach can be identified with the ability to predict SA settings that will lead to the limit of knock intensity based on indicating data referring to non-knocking conditions ( $\mathrm{SA}<\mathrm{KLSA})$. The stability of the estimated KLSA as SA increases is another quality factor.

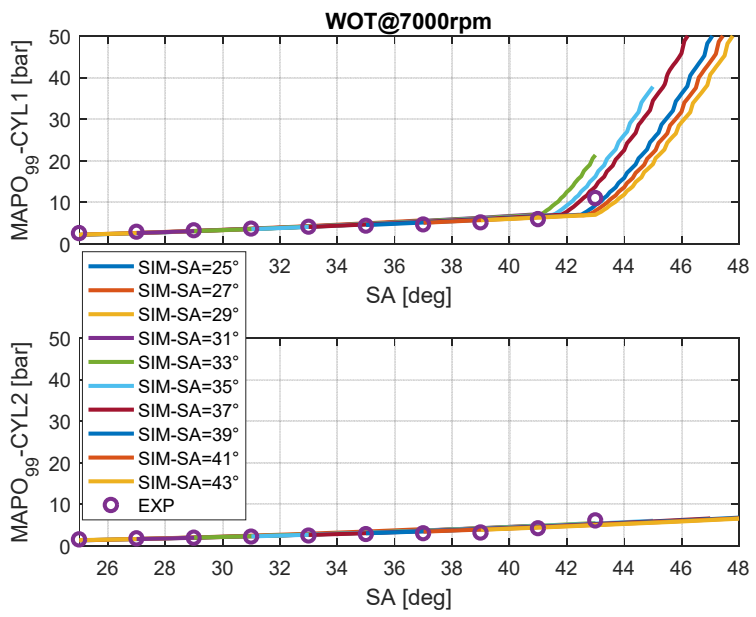

Page 10 of 15 

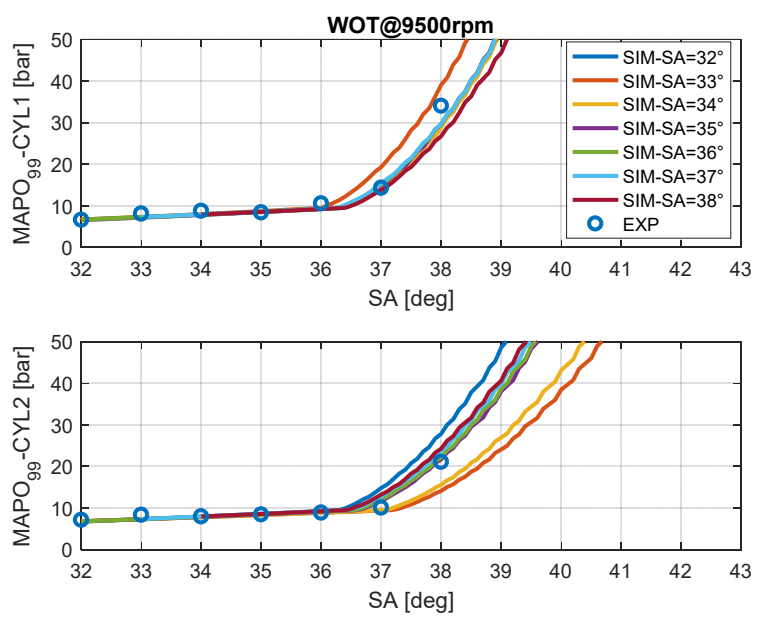

Figure 11: MAPO99 evaluations carried out for different SA settings and operating conditions

Figure 11 gives and idea of how the knock intensity estimator predicts the evolution of MAPO99 as a function of SA, starting from non-knocking SA settings, up to heavy knocking conditions.

It is interesting to notice that, already from the lowest available SA settings, the prediction of the SA where knocking phenomena starts taking place is accurate. The prediction quality, however, generally improves as SA approaches the light knock condition. It is also important to observe that, even with the lowest SA setting ('first attempt' conditions) there is no trace of false alarm, thanks to the physical approach used for the extrapolation of the model inputs. The behaviour of the two cylinders is well represented, even when the sensitivity of knock intensity to SA is different (as it can be noticed in the lower plot of Figure 11).
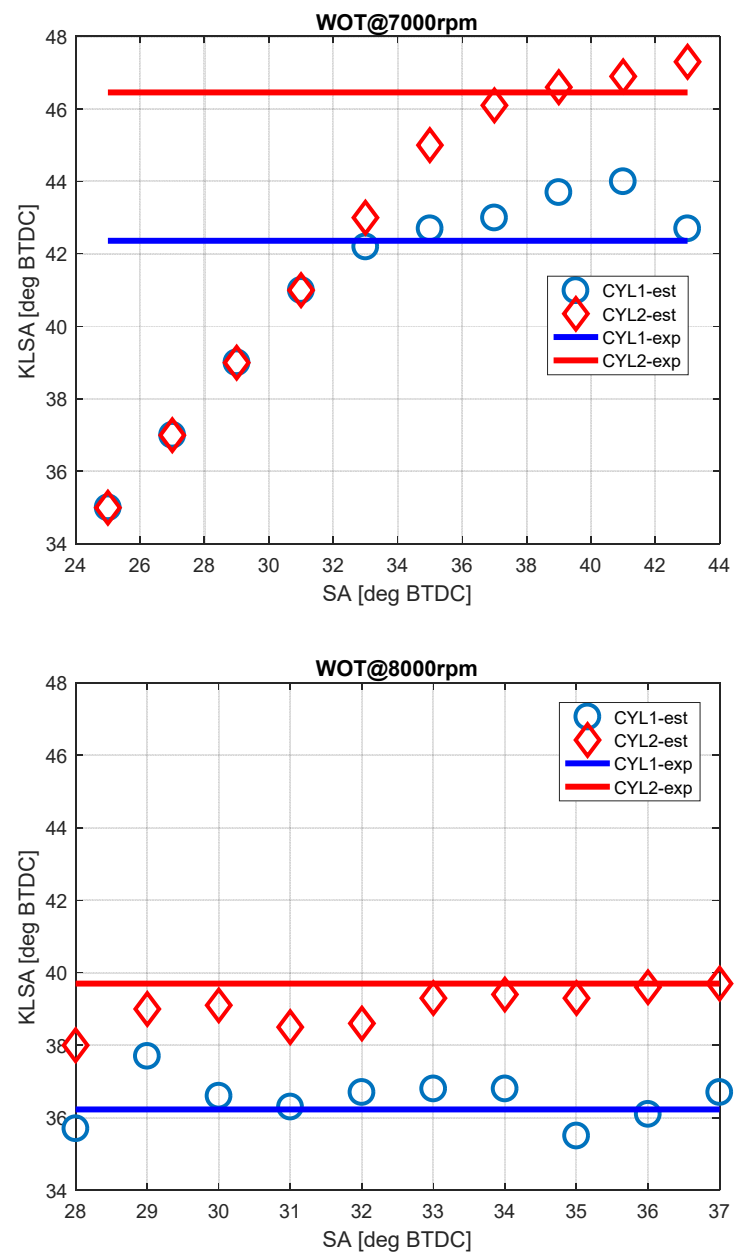

Page 11 of 15 


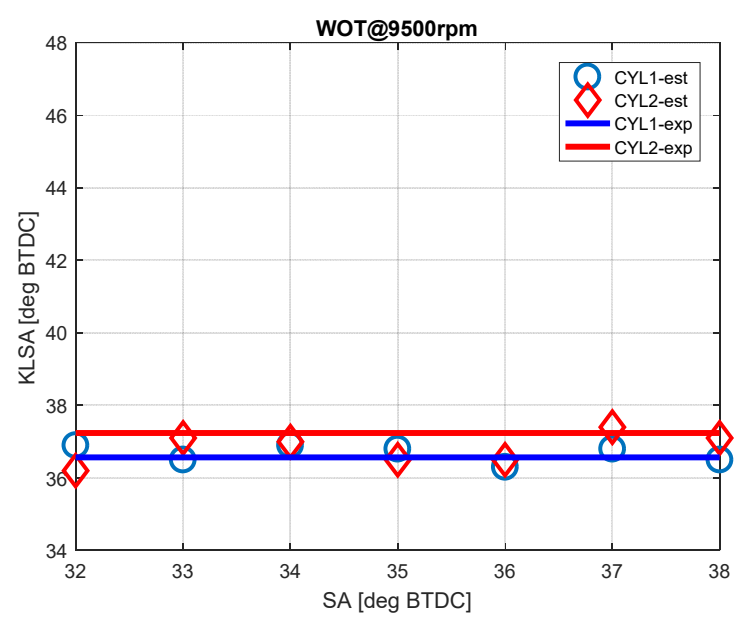

Figure 12: prediction of KLSA as a function of SA for different engine running conditions

Figure 12 shows the results of the knock intensity estimator in terms of KLSA prediction, during a Spark Advance sweep (starting from the lower SA value). The authority of the estimator has been limited to 10 crank angle degrees: in the upper plot the effect of this choice is observable in the first trend of KLSA with respect to SA.

When SA is low, the predicted KLSA is more than ten CA degrees higher than the actual SA, thus the prediction is saturated. As the difference between actuated SA and KLSA falls under 10 degrees, the predicted KLSA stabilizes. The good quality of the reconstruction is confirmed by the fact that, when the saturation is not applied to the predicted KLSA, the difference (ad its standard deviation) of the predicted value from the experimental one is small.

\section{Summary/Conclusions}

A simplified combustion model, fit for real-time Knock-Limited Spark Advance evaluation based on indicating data has been introduced: the model preserves the ability of describing the physical phenomena taking place during knocking combustions, although simplifications have been introduced to make it compatible with the use of cycle-based indicating data (namely, MFB10, MFB50).

The model has been calibrated, showing a good capability in predicting the average knock intensity, but also the scatter of knock indexes due to the temperature spatial distribution effect.

An approach to the on-line use of the model for the prediction of KLSA has been introduced: KLSA can be predicted based on indicating data referring to non-knocking conditions, using as inputs predicted normal distributions for MFB10, MFB50 and Intake Valve Closing Temperature. While for MFB the distribution characteristics (average value and standard deviation) can be inferred extrapolating indicating data sampled in nonknocking conditions, the temperature distribution standard deviation is kept constant, while the average value is changed in order to match the actual measured knock intensity. A simplified methodology to assess the statistical knock index (MAPO99) running the model only once for each SA setting has then been introduced, with the aim of lowering the computational power needed to predict knock intensity variation with SA.

The approach has been tested off-line on sampled indicating data that the Combustion Analysis System publishes on the CAN bus: since the methodology requires a limited computational power, a real-time implementation seems feasible. The results are interesting in terms of KLSA estimation accuracy and stability, even if the evaluation is carried out based on indicating data referring to SA settings far from KLSA.

The proposed methodology can be fitted to different type of engines, independently of the configuration, since it is based on the feedback of cylinder pressure signal, that refers to a single cylinder.

\section{References}

Jaesung Chung, Kyunghan Min, Seungsuk Oh, Myoungho Sunwoo, In-cylinder pressure based real-time combustion control for reduction of combustion dispersions in light-duty diesel engines, Applied Thermal Engineering, Volume 99, 25 April 2016, Pages 1183-1189, ISSN 1359-4311, http://dx.doi.org/10.1016/j.applthermaleng.2016.01.012.

Finesso, R., Marello, O., Misul, D., Spessa, E. et al., "Development and Assessment of Pressure-Based and Model-Based Techniques for the MFB50 Control of a Euro VI 3.0L Diesel Engine," SAE Int. J. Engines 10(4):2017, doi:10.4271/2017-01-0794.

Husted, H., Kruger, D., Fattic, G., Ripley, G. et al., "Cylinder Pressure-Based Control of Pre-Mixed Diesel Combustion," SAE Technical Paper 200701-0773, 2007, doi:10.4271/2007-01-0773. 
Schiefer, D., Maennel, R., and Nardoni, W., "Advantages of Diesel Engine Control Using In-Cylinder Pressure Information for Closed Loop Control," SAE Technical Paper 2003-01-0364, 2003, doi:10.4271/2003-01-0364.

S. Leonhardt, N. Muller and R. Isermann, "Methods for engine supervision and control based on cylinder pressure information," in IEEE/ASME Transactions on Mechatronics, vol. 4, no. 3, pp. 235-245, Sep 1999. doi: $10.1109 / 3516.789682$

Yoon, M., Lee, K., Sunwoo, M., and Oh, B., "Cylinder Pressure Based Combustion Phasing Control of a CRDI Diesel Engine," SAE Technical Paper 2007-01-0772, 2007, doi:10.4271/2007-01-0772.

Olsson, J., Tunestål, P., and Johansson, B., "Closed-Loop Control of an HCCI Engine," SAE Technical Paper 2001-01-1031, 2001, doi:10.4271/2001-01-1031.

Bengtsson, J., Strandh, P., Johansson, R., Tunestål, P. and Johansson, B. (2004), Closed-loop combustion control of homogeneous charge compression ignition (HCCI) engine dynamics. Int. J. Adapt. Control Signal Process., 18: 167-179. doi:10.1002/acs.788

G. M. Shaver, J. C. Gerdes and M. Roelle, "Physics-based closed-loop control of phasing, peak pressure and work output in HCCI engines utilizing variable valve actuation," Proceedings of the 2004 American Control Conference, Boston, MA, USA, 2004, pp. 150-155 vol.1.

Doosje, E., Willems, F., and Baert, R., "Experimental Demonstration of RCCI in Heavy-Duty Engines using Diesel and Natural Gas," SAE Technical Paper 2014-01-1318, 2014, doi:10.4271/2014-01-1318.

A.P. Carlucci, D. Laforgia, S. Motz, R. Saracino, S.P. Wenzel, Advanced closed loop combustion control of a LTC diesel engine based on incylinder pressure signals, Energy Conversion and Management, Volume 77, January 2014, Pages 193-207, ISSN 0196-8904, http://dx.doi.org/10.1016/j.enconman.2013.08.054.

Kumar, R., Zheng, M., Asad, U., and Reader, G., "Heat Release Based Adaptive Control to Improve Low Temperature Diesel Engine Combustion," SAE Technical Paper 2007-01-0771, 2007, doi:10.4271/2007-01-0771.

Rose, K., Ariztegui, J., Cracknell, R., Dubois, T. et al., "Exploring a Gasoline Compression Ignition (GCI) Engine Concept," SAE Technical Paper 2013-01-0911, 2013, doi:10.4271/2013-01-0911.

Sellnau, M., Foster, M., Moore, W., Sinnamon, J. et al., "Second Generation GDCI Multi-Cylinder Engine for High Fuel Efficiency and US Tier 3 Emissions," SAE Int. J. Engines 9(2):1002-1020, 2016, doi:10.4271/2016-01-0760.

Mladek, M. and Onder, C., "A Model for the Estimation of Inducted Air Mass and the Residual Gas Fraction using Cylinder Pressure Measurements," SAE Technical Paper 2000-01-0958, 2000, doi:10.4271/2000-01-0958.

Colin, G., Giansetti, P., Chamaillard, Y., and Higelin, P., "In-Cylinder Mass Estimation Using Cylinder Pressure," SAE Technical Paper 2007-240049, 2007, doi:10.4271/2007-24-0049.

Cavina N, Ponti F., "AFR estimation using in-cylinder pressure frequency analysis", ASME JOURNAL OF ENGINEERING FOR GAS TURBINES AND POWER, 2003, vol. 125, p. 812-819, ISSN: 0742-4795, doi: 10.1115/1.1563242..

Müller, N. and Isermann, R., "Control of Mixture Composition Using Cylinder Pressure Sensors," SAE Technical Paper 2001-01-3382, 2001, doi:10.4271/2001-01-3382.

Willems, F., Doosje, E., Engels, F., and Seykens, X., "Cylinder Pressure-Based Control in Heavy-Duty EGR Diesel Engines Using a Virtual Heat Release and Emission Sensor," SAE Technical Paper 2010-01-0564, 2010, doi:10.4271/2010-01-0564.

FIA delegate reports, e.g., https://www.fia.com/file/37249/download?token=Ltg3ifXa.

Tatsch, G., Martins, M., Lanzanova, T., Sari, R. et al., "Cylinder Pressure Based Engine Calibration of a Formula SAE Racing Engine," SAE Technical Paper 2014-36-0350, 2014, doi:10.4271/2014-36-0350.

G. Patterson, A Technique for Processing Cylinder Pressure and Test Bed Data Sets for Engine Speed Sweep Tests to Allow Reduced Testing Time with Enhanced Interpretation of Results, presented at 2008 SAE Motorsports Engineering Conference, SAE 2008-01-3006, 2008.

Xudong Zhen, Yang Wang, Shuaiqing Xu, Yongsheng Zhu, Chengjun Tao, Tao Xu, Mingzhi Song, The engine knock analysis - An overview, Applied Energy, Volume 92, April 2012, Pages 628-636, ISSN 0306-2619, http://dx.doi.org/10.1016/j.apenergy.2011.11.079.

Lorella Ceschini, Andrea Morri, Eleonora Balducci, Nicolò Cavina, Nahuel Rojo, Lucio Calogero, Luca Poggio, Experimental observations of engine piston damage induced by knocking combustion, Materials \& Design, Volume 114, 15 January 2017, Pages 312-325, ISSN 0264-1275, http://dx.doi.org/10.1016/j.matdes.2016.11.015.

Page 13 of 15 
Di Gioia, R., Papaleo, D., Vicchi, F., and Cavina, N., "Virtual GDI Engine as a Tool for Model-Based Calibration," SAE Technical Paper 2012-011679, 2012, doi:10.4271/2012-01-1679.

X. Yang and G. G. Zhu, "A mixed mean-value and crank-based model of a dual-stage turbocharged SI engine for Hardware-In-the-Loop simulation," Proceedings of the 2010 American Control Conference, Baltimore, MD, 2010, pp. 3791-3796.

doi: 10.1109/ACC.2010.5531459

Ponti, F., De Cesare, M., and Ravaglioli, V., "Development and Validation of a Methodology for Real-Time Evaluation of Cylinder by Cylinder Torque Production Non-Uniformities," SAE Technical Paper 2011-24-0145, 2011, doi:10.4271/2011-24-0145

Ponti, F., Ravaglioli, V., Moro, D., Serra, G., “MFB50 on-board estimation methodology for combustion control”, 2013, Control Engineering Practice, Volume 21 Issue 12, pp. 1821-1829, DOI: 10.1016/j.conengprac.2013.05.001

Ponti, F., Ravaglioli, V., Moro, D., Serra, G., "MFB50 on-board estimation methodology for combustion control”, 2013, Control Engineering Practice, Volume 21 Issue 12, pp. 1821-1829, DOI: 10.1016/j.conengprac.2013.05.001

Worret, R., Bernhardt, S., Schwarz, F., and Spicher, U., "Application of Different Cylinder Pressure Based Knock Detection Methods in Spark Ignition Engines,” SAE Technical Paper 2002-01-1668, 2002, doi:10.4271/200201-1668

Bozza, F., De Bellis, V., Minarelli, F., and Cacciatore, D., "Knock and Cycle by Cycle Analysis of a High Performance V12 Spark Ignition Engine. Part 2: 1D Combustion and Knock Modeling," SAE Int. J. Engines 8(5):2015, doi:10.4271/2015-24-2393

Livengood, J. C. and Wu, P. C., "Correlation of Autoignition Phenomenon in Internal Combustion Engines and Rapid Compression Machines", Fifth Symposium (International) on Combustion, pp. 347-356, 1955.

Millo, F., Rolando, L., Pautasso, E., and Servetto, E., "A Methodology to Mimic Cycle to Cycle Variations and to Predict Knock Occurrence through Numerical Simulation," SAE Technical Paper 2014-01-1070, 2014, doi:10.4271/2014-011070.

Corti, E., Forte, C., Cazzoli, G., Moro, D., Falfari, S., Ravaglioli, V., "Comparison of Knock Indexes Based on CFD Analysis", Energy Procedia, Volume 101, 2016, Pages 917-924, ISSN 1876-6102, http://dx.doi.org/10.1016/j.egypro.2016.11.116.

Corti, E., Forte, C., Bianchi, G., and Moro, D., "Relating Knocking Combustions Effects to Measurable Data," SAE Int. J. Engines 8(5):2015, doi: $10.4271 / 2015-24-2429$.

Mehl, M., Faravelli, T., Ranzi, E., Lucchini, T. et al., "Kinetic Modeling of Knock Properties in Internal Combustion Engines," SAE Technical Paper 2006-01-3239, 2006, doi:10.4271/2006-01-3239

Guezennec, Y. and Hamama, W., "Two-Zone Heat Release Analysis of Combustion Data and Calibration of Heat Transfer Correlation in an I. C. Engine," SAE Technical Paper 1999-01-0218, 1999, doi:10.4271/1999-01-0218

Forte, C., Corti, E., Bianchi, G., Falfari, S. et al., "A RANS CFD 3D Methodology for the Evaluation of the Effects of Cycle By Cycle Variation on Knock Tendency of a High Performance Spark Ignition Engine," SAE Technical Paper 2014-01-1223, 2014, doi:10.4271/2014-01-1223.

D'Adamo, A., Breda, S., Fontanesi, S., and Cantore, G., "A RANS-Based CFD Model to Predict the Statistical Occurrence of Knock in SparkIgnition Engines," SAE Int. J. Engines 9(1):2016, doi:10.4271/2016-01-0581

Leppard W. R., Individual-Cylinder Knock Occurrence and Intensity in Multi-Cylinder Engines, SAE Techinical paper, 820074, 1982.

Kim, K. and Ghandhi, J., “A Simple Model of Cyclic Variation,” SAE Technical Paper 2012-32-0003, 2012, doi:10.4271/2012-32-0003.

Dai, W., Trigui, N., and Lu, Y., "Modeling of Cyclic Variations in Spark-Ignition Engines,” SAE Technical Paper 2000-012036, 2000, doi:10.4271/2000-01-2036.

Vitek, O., Macek, J., Poetsch, C., and Tatschl, R., "Modeling Cycle-to-Cycle Variations in 0-D/1-D Simulation by Means of Combustion Model Parameter Perturbations based on Statistics of Cycle-Resolved Data", SAE Int. J. Engines 6(2):1075-1098, 2013, doi:10.4271/2013-01-1314.

Tatschl, R., Bogensperger, M., Pavlovic, Z., Priesching, P. et al., "LES Simulation of Flame Propagation in a Direct- Injection SI-Engine to Identify the Causes of Cycle-to- Cycle Combustion Variations," SAE Technical Paper 201301-1084, 2013, doi:10.4271/2013-01-1084.

Forte, C., Corti, E., Bianchi, G., Falfari, S. et al., "A RANS CFD 3D Methodology for the Evaluation of the Effects of Cycle By Cycle Variation on Knock Tendency of a High Performance Spark Ignition Engine," SAE Technical Paper 2014-01-1223, 2014, doi:10.4271/2014-01-1223.

\section{Contact Information}

Page 14 of 15 


\section{Prof. Enrico Corti}

E-mail address: enrico.corti2@unibo.it

Mail address: Dipartimento di Ingegneria Industriale (DIN), viale Risorgimento, 2, 40036 Bologna (Italy).

\section{Definitions/Abbreviations}

$\begin{array}{ll}\text { CAS } & \text { Combustion Analysis System } \\ \text { CHR } & \text { Cumulative Heat Release } \\ \text { IVC } & \text { Intake Valve Closing } \\ & \end{array}$

MAPO Maximum Amplitude of Pressure Oscillations

MFBxx Crank Angle corresponding to the Mass Fraction Burned xx

ROHR Rate Of Heat Release

SA Spark Advance

WOT Wide Open Throttle

cp Constant pressure specific heat capacity

cv Constant volume specific heat capacity

$\mathrm{k} \quad \mathrm{cp} / \mathrm{cv}$ ratio

$\mathrm{Kn}_{\text {trac }} \quad$ Knock tracer

Knindex $\quad$ Knock index

M Mass trapped in the cylinder

p Cylinder pressure

P Percentile of

p filt High-pass filtered cylinder pressure

$\mathrm{T}_{\mathrm{u}}, \mathrm{T}_{\mathrm{w}} \quad$ Unburned gas, wall temperatures

t time

$\mathrm{V}, \mathrm{Vu} \quad$ Cylinder, unburned gas volume

$\mathrm{xb} \quad$ Burned mass fraction

$\Delta \mathrm{p}_{\mathrm{knock}} \quad$ Pressure variation related to knock

$\theta, \theta_{\mathrm{s}}, \theta_{\mathrm{e}} \quad$ Crank angle (start/end for knock intensity evaluation) 